\title{
Management of a Giant Uterine Leiomyoma
}

Lim PT ${ }^{1}$, Tan $\mathrm{CH}^{2}$, Qi ML ${ }^{1}$, Wright $\mathrm{A}^{1}$, Wong WL ${ }^{1}$, Chia $\mathrm{HL}^{3}$, Phoon JWL

1. Department of Obstetrics and Gynaecology, KK Women's and Children's Hospital, Singapore

2. Department of Anaesthesiology, KK Women's and Children's Hospital, Singapore

3. Department of Plastics, Reconstructive and Aesthetic Surgery, KK Women's and Children's Hospital, Singapore

\section{Our Case}

A 53 year old post menopausal malay woman, virgo intacta, presented with orthopnea, exertional dyspnea and a massive abdominal mass for many years limiting mobility. Physical examination revealed a cachexic lady with a tense abdomen and a large palpable mass extending up to the xiphisternum with prominent overlying vessels.(Fig 1) There was reduced air entry in both lung bases.

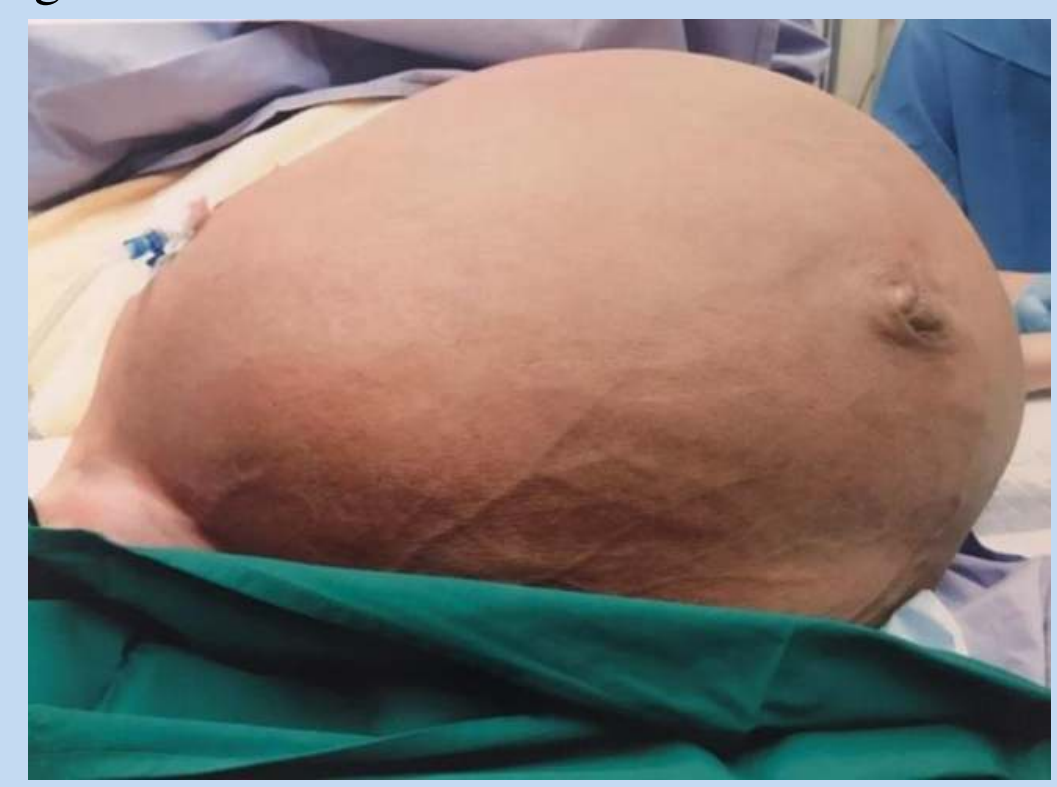

Fig1. Massive abdominal mass extending to xiphis sternum with prominent overlying vessels

\section{Investigations}

- CT Abdomen-Pelvis: Large multilobulated solid mass with cystic components occupying most of the abdominal and pelvic cavity measuring $47.4 \mathrm{~cm}$. Gross ascites was noted and there were no hepatic lesions or enlarged lymph nodes.

- Transthoracic echocardiogram: Ejection fraction of $61 \%$ with no regional wall abnormalities.

- Lung function test showed restrictive lung disease.

- CT pulmonary aortogram: no pulmonary embolism.

Haemoglobin: 12g/dL. Platelets: 406x109/L. LDH: 677U/L

\section{Treatment}

The patient was reviewed by the anaesthesia team and respiratory medicine physician for optimisation of pre-operative function. She also underwent an ultrasound guided drainage of ascites pre-operatively for temporary relief of respiratory symptoms and to improve respiratory function.

A total abdominal hysterectomy, bilateral salpingo-oophorectomy with frozen section was then performed by the gynaecology and plastic surgery teams. Intra-operative findings revealed a pelvic mass which was multilobulated and densely adherent to the anterior abdominal wall and peritoneum with multiple feeding vessels. Extensive adhesiolysis was done to free the mass and the fibroid was decapsulated for ease of removal. Frozen section confirmed a benign leiomyoma. The final excised specimen measured $64 \times 50.5 \times 15 \mathrm{~cm}$ and weighed $27.8 \mathrm{~kg}$. (Fig 2)

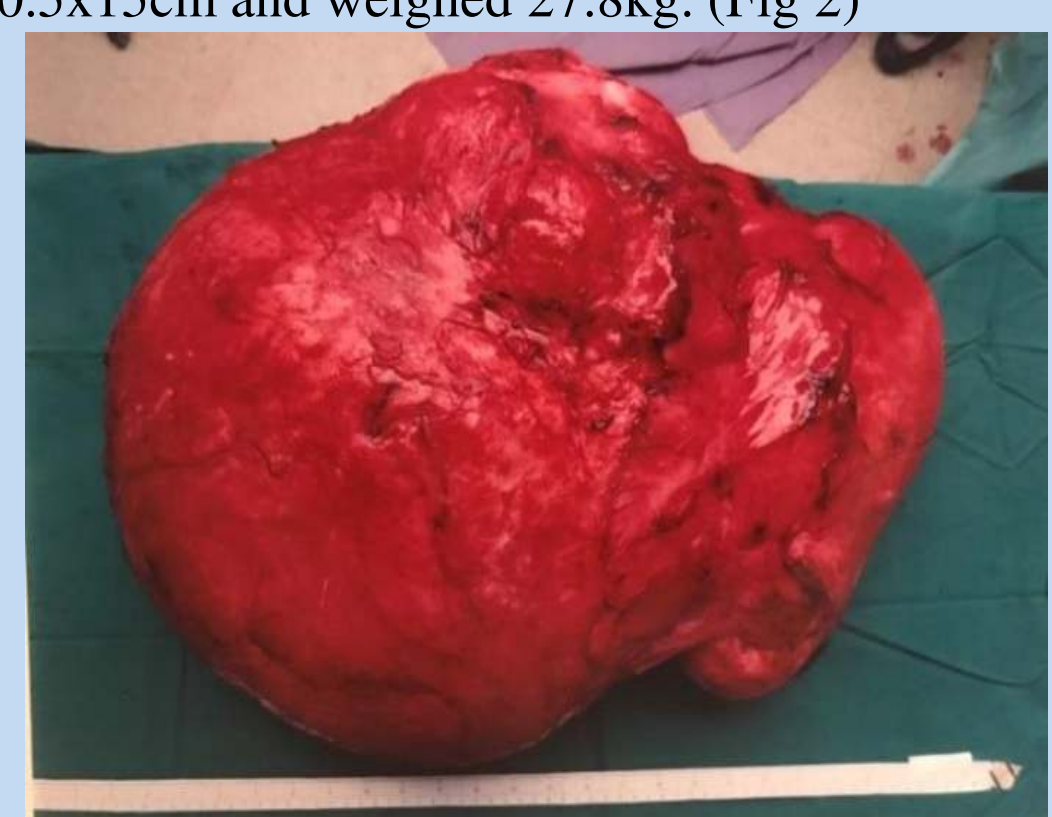

Plastic surgeons performed a Fleur-de-Lys pattern excision and closure to address the horizontal and vertical laxity. [Fig 3]

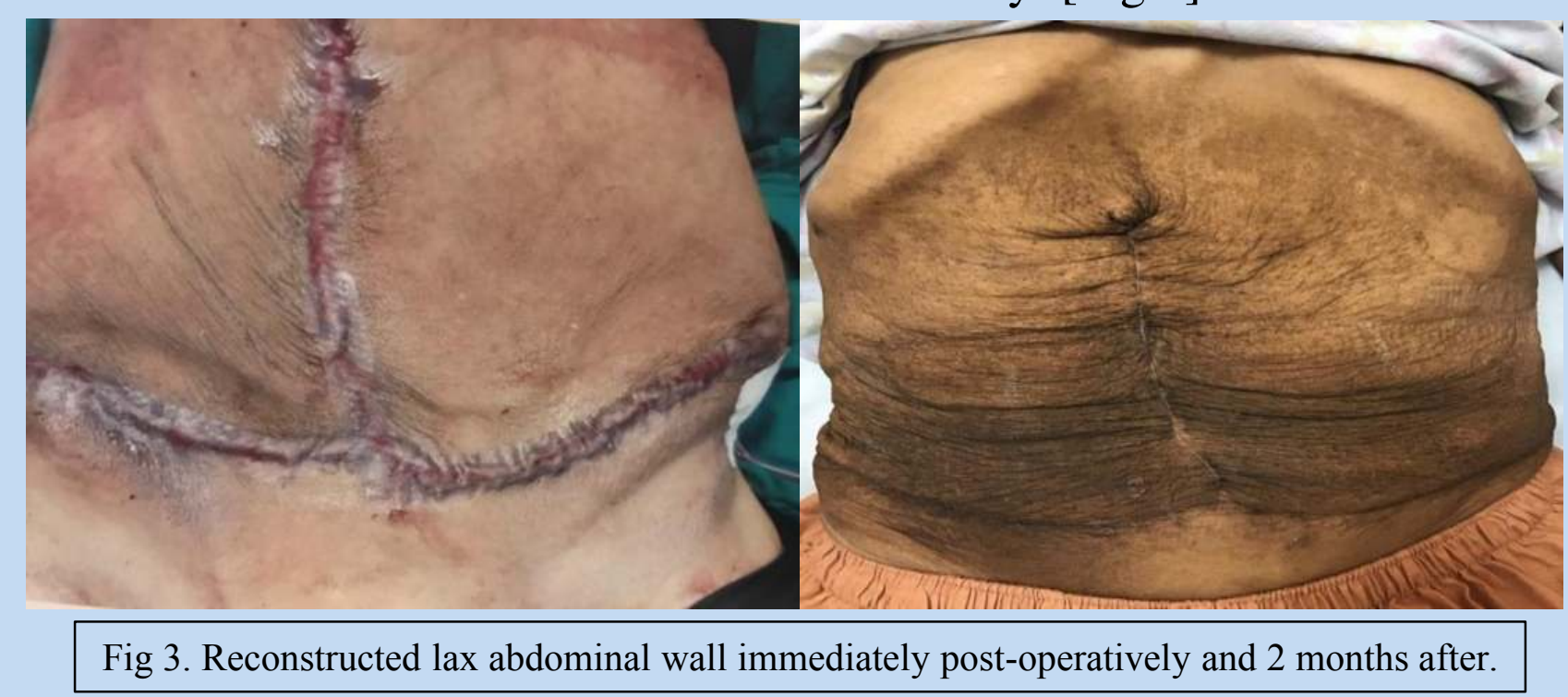

Intra-operative blood loss was 7 litres, requiring activation of the hospital's Massive Transfusion Protocol. A total of 4.1L of packed cells, 1.1L of fresh frozen plasma, $302 \mathrm{ml}$ cryoprecipitate and $8 \mathrm{~L}$ of crystalloids was transfused.

Post-operatively, she was admitted to the Intensive Care Unit for close monitoring. Her recovery was complicated by haemorrhagic shock and coagulopathy requiring inotropic support. She was extubated and weaned off inotropes on post-operative day 3 . She was stable and discharged on postoperative day 7.

\section{Discussion}

Giant uterine leiomyoma is defined as weighing $11.4 \mathrm{~kg}$ or more ${ }^{1}$ The largest uterine tumour ever removed from a patient who survived a procedure weighed $45.4 \mathrm{~kg}$ and was reportedly the $34^{\text {th }}$ largest tumour weighing more than $18.2 \mathrm{~kg}$ since $1878 .{ }^{1}$ The average growth rate of fibroids is $9 \%$ over six months ${ }^{2}$. In postmenopausal woman, the incidence of sarcoma is $1-2 \%$ and they have a rapid doubling time of four weeks. ${ }^{3}$

In 1933, Owings et al published a series of patients with surgical excisions of large uterine leiomyoma and concluded an operative death rate of $14.8 \%{ }^{4}$

The National Massive Transfusion Protocol is a centralised systematic clinical workflow implemented by the National Blood Services Group initially created for military trauma settings. It allows accelerated delivery and transfusion of blood components in a ratio of 1:1:1 (packed red blood cells: whole blood-derived platelets: fresh frozen plasma). ${ }^{5}$

\section{Conclusion}

- Pre-operative optimisation of patient function and work up of comorbidities allow for better preparation and anticipation of potential surgical difficulties and complications.

- Close monitoring of intra-operative haemodynamic status with prompt replacement of fluid losses is critical.

- Post-operative development of haemorrhagic shock and coagulopathy are common and should be treated accordingly.

- Multidisciplinary care in the management of complicated surgical patients is essential in optimisation of surgical outcomes.

. Evans 3rd AT, Pratt JH. A giant fibroid uterus. Obstetrics and gynecology. 1979 Sep;54(3):385-6

2. Peddada SD, Laughlin SK, Miner K, Guyon JP, Haneke K, Vahdat HL, Semelka RC, Kowalik A, Armao D, Davis B, Baird DD. Growth of uterine leiomyomata among premenopausal black and white women. Proceedings of the National Academy of Sciences. 2008 Dec 16;105(50):19887-92

3. Vellanki VS, Rao M, Sunkavalli CB, Chinamotu RN, Kaja S. A rare case of uterine leiomyosarcoma: a case report. Journal of medical case reports. $2010 \mathrm{Jul}$ 22;4(1):222

4. Owings JC. Fibromyoma of the uterus: Report of a case of sixty-five pound solid fibromyoma, with a review of large cystic and solid uterine myomas. Archives of Surgery. 1933 Nov 1;27(5):897-904

5. Chay J, Koh M, Tan HH, Ng J, Ng HJ, Chia N, Kuperan P, Tan J, Lew E, Tan LK, Koh PL. A national common massive transfusion protocol (MTP) is a feasible and advantageous option for centralized blood services and hospitals. Vox sanguinis. 2016 